\title{
Benign hyperfiltration after living kidney donation
}

\author{
Roland C. Blantz ${ }^{1,2}$ and Robert W. Steiner ${ }^{1}$ \\ 'Department of Medicine, School of Medicine, Division of Nephrology-Hypertension, UCSD, San Diego, California, USA. Veterans Affairs San Diego Healthcare System, San Diego, California, USA.
}

\begin{abstract}
Almost one-third of transplanted kidneys come from living donors, who sacrifice approximately $30 \%$ of their pre-donation glomerular filtration rate (GFR) after they experience compensatory hypertrophy and hyperfiltration in their remaining kidney. Although hyperfiltration can cause glomerular injury, many studies have suggested that donor nephrectomy itself does not cause long-term loss of GFR at a higher rate than what is seen in the normal aging population. However, when post-donation kidney diseases occur in an unfortunate few, recent studies suggest that GFR loss at donor nephrectomy increases the risk of eventual end-stage renal disease (ESRD). In this issue of the $J C l$, Lenihan and colleagues evaluated glomerular dynamics in a cohort of kidney donors prior to, within 1 year of, and several years after kidney donation. Their results suggest that adaptive hyperfiltration in the remaining kidney occurs without glomerular hypertension, furthering our understanding of the relatively benign renal outcomes for most living kidney donors.
\end{abstract}

\section{Altered kidney function in living donors}

Several longstanding issues associated with living kidney donation are currently being reexamined. In the early days, centers were concerned about several possible long-term risks to kidney donors (1). As nephrectomy reduces a donor's glomerular filtration rate (GFR) by approximately $30 \%$, a "uremic" effect was viewed as a potential threat to donor health; centers were also concerned that hyperfiltration in the remaining kidney might worsen the progression of new-onset post-donation kidney diseases, should they arise; most important, animal models suggested that single-nephron hyperfiltration itself might cause glomerular sclerosis, threatening the possibility of widespread, accelerated losses of GFR after donation (1). In these models, both single-nephron GFR and glomerular capillary pressure were increased. Although the individual contributions of each of these two factors were not determined, it is widely suspected that increased glomerular pressure contributes to kidney damage (2).

\section{Analysis of the post-donation glomerular response}

In this issue, Lenihan and colleagues report on a longitudinal study of glomerular dynamics in living kidney donors prior to, within 1 year of, and a median of 6.3 years after kidney donation (3). This study is a valuable effort to characterize the increase in post-nephrectomy single-nephron GFR in the years following kidney donation. GFR is dictated by four determinants of glomerular ultrafiltration: renal plasma flow (RPF); systemic oncotic pressure $(\pi \mathrm{A})$; the hydraulic pressure gradient $(\Delta \mathrm{P})$; and the glomerular ultrafiltration coefficient $\left(\mathrm{K}_{\mathrm{f}}\right)$, which is a product of the hydraulic conductivity of the glomerular capillary membrane and glomerular surface area (4). Lenihan et al. directly measured two of these four determinants, $\mathrm{RPF}$ and $\pi \mathrm{A}$, while the individual contributions of $\mathrm{K}_{\mathrm{f}}$ and $\Delta \mathrm{P}$ were derived

Related Article: p. 1311

Conflict of interest: The authors have declared that no conflict of interest exists

Reference information: / Clin Invest. 2015;125(3):972-974. doi:10.1172/JCI80818.

from detailed equations and plausible assumptions regarding glomerular hemodynamics. The authors also measured a $40 \%$ increase in GFR in the remaining, untransplanted donor kidney (3).

The four determinants of GFR are not truly independent variables. Micropuncture assessments of renal function under a variety of physiologic and pathophysiologic conditions have shown important interdependencies among them (5). For example, $\pi \mathrm{A}$ and $\Delta \mathrm{P}$ vary directly and primarily as a result of changes in systemic protein concentration, which confers considerable stability to ultrafiltration pressure $\left(\mathrm{P}_{\mathrm{UF}}\right)$, which is the difference between $\Delta \mathrm{P}$ and $\pi \mathrm{A}\left(\mathrm{P}_{\mathrm{UF}}=\Delta \mathrm{P}-\pi \mathrm{A}\right)(4,6)$. Previous studies have clearly demonstrated an inverse, bidirectional relationship between $\Delta \mathrm{P}$ and $\mathrm{K}_{\mathrm{f}}(6)$. For example, if $\mathrm{K}_{\mathrm{f}}$ is acutely reduced or chronically reduced, such as occurs after immune injury to the glomerulus, $\Delta \mathrm{P}$ clearly increases $(7,8)$. A reduction in a conductance pathway $\left(\mathrm{K}_{\mathrm{f}}\right)$ for water and small solutes should logically increase upstream hydraulic pressure. Alternatively, when $\Delta \mathrm{P}$ is increased in the glomerulus over time, such as occurs in a remnant kidney or following five-sixths nephrectomy $(2,9), \mathrm{K}_{\mathrm{f}}$ tends to markedly decrease either via loss of hydraulic conductivity or of glomerular surface area (5); however, it is not clear mechanistically how or why this occurs. The reduction of $\mathrm{K}_{\mathrm{f}}$ in response to increases in hydraulic pressure has been clearly demonstrated in experimental rodent models $(2,9)$. Such an increase in $\Delta \mathrm{P}$ might reduce $\mathrm{K}_{\mathrm{f}}$ and lead to long-term kidney dysfunction in kidney donors.

Under certain conditions, the normal rat demonstrates filtration pressure equilibrium, the point at which filtration ceases because hydraulic pressure is exactly opposed by increased oncotic pressure along an idealized glomerular capillary $(4,5)$. Whether this is also seen in humans is of central importance in two respects. First, unique $\mathrm{K}_{\mathrm{f}}$ values can be assigned only if filtration pressure equilibrium is not achieved (10), a point not 
addressed by Lenihan et al. (3). At filtration pressure equilibrium, only minimal estimates of $\mathrm{K}_{\mathrm{f}}$ can be computed. Second, the presence or absence of filtration pressure equilibrium strongly modifies the effects of increased RPF on GFR and filtration fraction (4). In the kidney donors evaluated by Lenihan and colleagues, RPF and GFR both increased by $40 \%$, maintaining a constant filtration fraction of about 22\%. Lenihan and colleagues argue in favor of filtration pressure disequilibrium in human donors, leading them to conclude that $\mathrm{K}_{\mathrm{f}}$ must have increased in parallel with RPF in order for GFR to increase proportionately (3). Lenihan et al. also allow that if filtration pressure equilibrium occurred in the donors studied, increased RPF could increase GFR without a simultaneous increase in $\mathrm{K}_{\mathrm{f}}(4,5)$; however, they argue that this scenario is unlikely. Lenihan and colleagues are to be congratulated for recognizing that some combination of increased $\Delta \mathrm{P}$ and lesser increases in $\mathrm{K}_{\mathrm{f}}$ might satisfy the conditions they observed in their study (3). They show that a modest increase in $\Delta \mathrm{P}$ of approximately 3 to $4 \mathrm{mmHg}$ would permit $\mathrm{K}_{\mathrm{f}}$ to remain constant. The most extreme alternative hypothesis requires $\Delta \mathrm{P}$ to increase to 45 to $50 \mathrm{mmHg}$, with a reciprocal $30 \%-45 \%$ decrease of $\mathrm{K}_{\mathrm{f}}$, to still satisfy the functional findings in this study; however, given the measured increase in renal cortical volume, Lenihan and colleagues conclude that the most likely explanation is a parallel increase of $\mathrm{RPF}$ and $\mathrm{K}_{\mathrm{f}}$, with $\triangle \mathrm{P}$ remaining constant. This finding is consistent with developmental kidney growth in normal rodents, whereby GFR increases with kidney size via nearly identical increases in RPF and $\mathrm{K}_{\mathrm{f}}$, while $\Delta \mathrm{P}$ remains constant (11). Lenihan et al. also recognize the possibility of heterogeneity of $\Delta \mathrm{P}$ and $\mathrm{K}_{\mathrm{f}}$ responses among kidney donors that still satisfy their whole-organ cohort data on GFR, $\mathrm{RPF}$, and filtration fraction and leave open the possibility that certain living donor populations may be at risk for future kidney dysfunction as a consequence of "hyperbaric" glomerular pressure.

\section{Clinical implications}

For decades, the additional, incremental long-term renal risk of kidney donation has been thought to be minimal or nil
(12). Many studies found that in the first 10-15 years after donation, kidney donors appeared to stay healthy, with little ESRD and stable GFRs. The need to separately address the risks of "uremia," new-onset kidney diseases, and adaptive hyperfiltration was not pressing or obvious. Indeed, some single-point analyses have indicated that post-donation GFR actually increases slightly in donors over time, rather than declining slowly, as it does in normal individuals within the general population. However, in one major study, such singlepoint data conflicted with actual decrements of about $6 \mathrm{ml} / \mathrm{min} / 1.73 \mathrm{~m}^{2} /$ decade when the GFR was directly measured twice over several years (13). The current study by Lenihan and colleagues (3) is consistent with these largely benign post-donation outcomes. Specifically, their results suggest that hyperfiltration following kidney donation does not damage the glomerulus by increasing glomerular hydraulic pressure.

Lenihan and colleagues are careful not to claim that benign post-donation glomerular adaptation supports a categorical "no-risk" view of living kidney donation. They cite two recently published studies that suggest that kidney donors are at a substantially increased risk of ESRD when compared with that of appropriately selected individuals with both kidneys as controls $(14,15)$. In these studies, the overall absolute rate of post-donation ESRD was very low, but the risk of ESRD in donors was about 8 to 11 times higher than that in controls during their respective 7.6and 15.1-year observation periods. Expert commentary has criticized the composition of the control groups, the statistical analysis $(16,17)$, the attempt to derive a single-risk estimate to cover what may be markedly heterogeneous individual risks, and the extrapolation of predictably low 10- to 15-year ESRD risks to lifetime outcomes (18). That same commentary attributed at least some of the increase in the relative risk of kidney donors for ESRD to GFR loss at donor nephrectomy. All else being equal, since donors would have, on average, 30\% less "renal reserve" when progressive kidney diseases arose, over a given study interval, they would reach ESRD well before their two kidney comparators $(16,18)$. In other words, for all donors, lower post-donation GFRs are important renal risk factors (18).
These recent studies have also demonstrated that current donor selection protocols may not be able to exclude all donor candidates who will develop future kidney diseases, a risk that is distinct from the risks of donor nephrectomy per se (18). Young donors who are fated to develop these diseases will be particularly difficult to identify, as they may well be normal at the pre-donation medical examination $(18,19)$. Twenty-five-year-old candidates, for example, will be several decades away from their highest-risk years for ESRD, as only $13 \%$ of ESRD occurs by age 44 each year, and about $50 \%$ of cases occur after age 64 (20). They will be at greatest risk for diabetic ESRD, which will not appear until diabetes has been present for at least 20 years (18). For those young donors, in whom progressive post-donation kidney diseases may not begin until middle age, preservation of their interim "renal reserve" is particularly important. The apparently benign physiology of adaptive post-donation GFR in this way helps limit the ESRD risks for both the few donors who will develop post-donation kidney diseases as well as for all the others who will not. This study by Lenihan et al. is a valuable contribution to understanding those outcomes.

\section{Acknowledgments}

R.C. Blantz received funding support from the NIH (R01 DK28602 and P30 DK079337) and the Research Service of the Department of Veterans Affairs.

Address correspondence to: Roland C. Blantz, UCSD and VASDHS, 3350 La Jolla Village Drive - 9111-H San Diego, California 92161-002, USA. Phone: 858.552.8585, ext.1146; E-mail: rblantz@ucd.edu.

1. Ogden DA. Consequences of renal donation in man. Am J Kidney Dis. 1983;2(5):501-511.

2. Hostetter TH, Olson JL, Rennke HG, Venkatachalam MA, Brenner BM. Hyperfiltration in remnant $t$ nephrons: a potentially adverse response to renal ablation. Am J Physiol. 1981;241(1):F85-F93.

3. Lenihan CR, et al. Longitudinal study of living kidney donor glomerular dynamics after nephrectomy. JClin Invest. 2015;125(3):1311-1318.

4. Tucker BJ, Blantz RC. An analysis of the determinants of nephron filtration rate. Am J Physiol. 1977;232(6):F477-F483.

5. Tucker BJ, Blantz RC. Effects of glomerular filtration dynamics on the glomerular permeability coefficient. Am J Physiol.1981;240(3):F245-F254. 
6. Blantz RC, Rector FC Jr, Seldin DW. The effect of hyperoncotic albumin expansion upon glomerular ultrafiltration in the rat. Kidney Int. 1974;6(4):209-221.

7. Blantz RC, Wilson CB. Acute effects of antiglomerular basement membrane antibody on the process of glomerular filtration in the rat. JClin Invest. 1976;58(4):899-911.

8. Blantz RC, Tucker BJ, Wilson CB. The acute effects of antiglomerular basement membrane antibody on the process of glomerular filtration in the rat. The influence of dose and complement depletion. JClin Invest. 1978;61(4):910-921.

9. Anderson S, Meyer TW, Rennke HG, Brenner BM. Control of glomerular hypertension limits glomerular injury in rats with reduced renal mass. JClin Invest. 1985;76(2):612-619.

10. Steiner RW, Tucker BJ, Blantz RC. Glomerular hemodynamics in rats with chronic sodium depletion. Effect of saralasin. JClin Invest. 1979;64(2):503-512.

11. Tucker BJ, Blantz RC. Factors determining superficial nephron filtration in the mature, growing rat. Am J Physiol. 1977;232(2):F97-F104.

12. Ramos E, Vijayan A, Vella J. Evaluation of the living kidney donor and risk of donor nephrectomy. UpToDate Web site. http://www.uptodate. com/contents/evaluation-of-the-living-kidneydonor-and-risk-of-donor-nephrectomy. Updated December 19, 2014. Accessed January 22, 2015.

13. Ibrahim HN, et al. Long-term consequences of kidney donation. NEngl JMed.2009;360(5):449-460.

14. Muzaale AD, et al. Risk of end-stage renal disease following live kidney donation. JAMA. 2014;311(6):579-586.

15. Mjoen G, et al. Long-term risks for kidney donors. Kidney Int. 2014;86(1):162-167.

16. Gill JS, Tonelli M. Understanding rare adverse outcomes following living kidney donation. JAMA. 2014;311(6):577-578.

17. Boudville N, Garg AX. End-stage renal disease in living kidney donors. Kidney Int. 2014;86(1):20-22.

18. Steiner R, Ix JH, Rifkin DE, Gert B. Estimating risks of de novo kidney diseases after living kidney donation. Am J Transplant. 2014;14(3):538-544.

19. Organ Procurement and Transplantation Network (OPTN). OPTN Organ Procurement and Transplantation Network Policies. Policy 14.3. Informed Consent Requirements. OPTN Web site. http:// optn.transplant.hrsa.gov/ContentDocuments/ OPTN_Policies.pdf. Updated February 2, 2015. Accessed February 3, 2015.

20. United States Renal Data System. 2014 Annual Data Report: Epidemiology of Kidney Disease in the United States. Bethesda, Maryland, USA: National Institutes of Health, National Institute of Diabetes and Digestive and Kidney Diseases; 2014. 